\title{
Application of the two-parameter single shooting method for natural vibration analysis of Euler- Bernoulli beams
}

\author{
Ryszard Hołubowski ${ }^{*}$ \\ Wroclaw University of Science and Technology, Faculty of Civil Engineering, Wroclaw, Poland
}

\begin{abstract}
The paper presents a new method of calculating the natural frequencies and modes of Euler-Bernoulli beams. The formulated algorithm is based on the fourth order homogeneous ordinary differential equation of motion that allows different support conditions to be considered. The idea of the method is to replace a two-point boundary value problem with an initial value problem. Distinct from the classic approach, where only one unknown parameter is considered, the method enables problems with two parameters to be solved. The proposed two-parameter version of the single shooting method is verified on the example of a non-prismatic simply supported beam and a prismatic propped cantilever beam resting on Winkler foundation and loaded by axial force. The obtained results confirm the correctness and high effectiveness of the presented method.
\end{abstract}

\section{Introduction}

Natural vibration analysis is one of the fundamental issues of structural dynamics. Knowledge of eigenfrequencies and eigenmodes allows structures to be protected against resonant states, structural parameters to be identified, and inter alia forced vibrations to be analysed. Therefore, the natural vibration problem is considered by many researchers. In this paper, an originally formulated two-parameter single shooting method has been used to compute the natural frequencies and modes of Euler-Bernoulli beams. The proposed method can be an alternative to the commonly used finite element method due the fact that the beam does not have to be divided into many elements to obtain accurate enough solution, even though the geometry of the beam is very complex.

The standard single shooting method is based on the second order ordinary differential equation and allows a two-point boundary value problem to be reduced to an initial one [1]. In the literature, we can find various modifications of the classical approach. El-Gebeily and Attili [2] proposed an iterative shooting method for a certain class of singular two-point boundary value problems. Pai and Palazotto [3] used a multiple shooting method in the large-deformation analysis of flexible beams. Ha [4] introduced a new shooting technique for non-linear boundary value problems. Ibrahim et al. [5] investigated large amplitude periodic forced vibrations of curved beams under periodic excitation using a modified shooting approach based on the Newmark time marching scheme and arc length

* Corresponding author: ryszard.holubowski@pwr.edu.pl 
continuation algorithm. Ribeiro [6] demonstrated an algorithm based on the shooting method to solve finite element equations of motion and to study geometrically non-linear vibrations of beams and plates.

\section{Two-parameter single shooting method}

The idea of the two-parameter single shooting method will be presented using the example of natural transverse vibrations of a Euler-Bernoulli beam. To obtain natural frequencies and modes, the following partial differential equation that describing the free vibration process is considered [7]

$$
\frac{\partial^{2}}{\partial x^{2}}\left[E I(x) \frac{\partial^{2} w(x, t)}{\partial x^{2}}\right]+\rho A(x) \frac{\partial^{2} w(x, t)}{\partial t^{2}}+\frac{\partial}{\partial x}\left[p(x) \frac{\partial w(x, t)}{\partial x}\right]+k(x) w(x, t)=0,
$$

where $E I(x)$ is the bending stiffness, $\rho A(x)$ represents the mass per unit length, $p(x)$ is the axial compressive force, and $k(x)$ describes the stiffness parameter of the Winkler foundation. The solution of Eq. (1) can be written in the form of separated variables

$$
w(x, t)=f(x) g(t) .
$$

Substituting formula (2) into Eq. (1) and introducing a constant $\omega^{2}$ leads to two ordinary differential equations

$$
\begin{gathered}
\ddot{g}(t)+\omega^{2} g(t)=0 \\
E I(x) f^{\prime \prime \prime \prime}(x)+2 E I^{\prime}(x) f^{\prime \prime \prime}(x)+\left[E I^{\prime \prime}(x)+p(x)\right] f^{\prime \prime}(x)+ \\
+p^{\prime}(x) f^{\prime}(x)+\left[k(x)-\omega^{2} \rho A(x)\right] f(x)=0 .
\end{gathered}
$$

where $\left({ }^{\cdot}\right)=d / d t$ and ()$^{\prime}=d / d x$.

The general solution of Eq. (3.1) has the harmonic form

$$
g(t)=C_{1} \sin \omega t+C_{2} \cos \omega t .
$$

Taking into account the following initial conditions

$$
g(0)=0 \text { and } \dot{g}(0)=\omega,
$$

we obtain the particular integral

$$
g(t)=\sin \omega t
$$

and the solution of Eq. (1) in the form

$$
w(x, t)=f(x) \sin \omega t,
$$

in which $\omega$ is the circular natural frequency and function $f(x)$ describes the corresponding natural mode. Substituting solution (7) into Eq. (1) gives the ordinary differential equation

$$
\frac{d^{2}}{d x^{2}}\left[E I(x) \frac{d^{2}}{d x^{2}} f(x)\right]-\omega^{2} \rho A(x) f(x)+\frac{d}{d x}\left[p(x) \frac{d}{d x} f(x)\right]+k(x) f(x)=0,
$$

which is a starting point for the shooting method. Despite the type of beam static scheme, the conditions imposed on the solution are always determined at different points of the interval of a solution's definition. In most cases, a two-point boundary value problem 
is considered. Usually, two conditions are specified at the beginning point and two at the end point of the beam. In order to apply one of the numerical integration methods to solve Eq. (8), all initial conditions have to be known. Therefore, the standard single shooting method is insufficient because it allows the replacement of only one condition, which is determined at the end, with the initial condition. In the considered case, apart from two conditions defined at the end, there is an additional parameter $\omega$, which is the sought unknown. The method proposed in this paper enables the raised problem to be solved.

The procedure of the method, explained on the example of beam natural transverse vibration, is as follows:

a) Formulate a fourth-order ordinary differential equation (8) with a natural frequency $\omega$ as the parameter and a solution $f(x)$ defined on an interval $[\mathrm{a}, \mathrm{b}]$.

b) Evaluate values of function $\eta_{1}(\omega, \alpha)=d^{k_{1}} f(b) / d x^{k_{1}}$ to create a first hit surface. The parameter $\alpha$ represents a value of one of two unknown initial conditions, whereas the value of the second one is freely determined due to the fact that every single mode can be arbitrarily normalizing. The order $k_{1}$ of the derivative depends on the boundary conditions.

c) Similar to the previous point, build a second hit surface $\eta_{2}(\omega, \alpha)=d^{k_{2}} f(b) / d x^{k_{2}}$ that is based on a second boundary condition.

d) Solve the system of two nonlinear algebraic equations using one of the iterative methods, like Newton's method, the secant method, or Brent's method

$$
\left\{\begin{array}{l}
\eta_{1}(\omega, \alpha)=0 \\
\eta_{2}(\omega, \alpha)=0
\end{array}\right.
$$

to find the pairs of parameters $(\omega, \alpha)$ that satisfy the boundary conditions at the endpoint. When the natural frequencies $\omega_{n}$ and the corresponding initial conditions $\alpha_{n}$ are known, we can return to differential equation (8) and solve it $n$ times, numerically obtaining $n$ natural modes.

\section{Numerical examples}

To verify the correctness and effectiveness of the two-parameter single shooting method two examples are taken into account: a non-prismatic simply supported concrete beam and a prismatic propped cantilever steel beam resting on the Winkler foundation and loaded by compressive force. All numerical analyses based on the proposed method were carried out using Mathematica software. The obtained results in the form of natural frequencies were compared with solutions computed by the commercial finite element program SOFiSTiK.

\subsection{Non-prismatic beam}

The simply supported beam with a span $L=5.0 \mathrm{~m}$, a rectangular cross-section of variable width $b(x)=0.247561+0.164911 x-0.00692905 x^{3}$ and a height $h(x)=0.8-0.1 x$ (in meters) is made of concrete C $25 / 30$ with a density of $\rho=2400 \mathrm{~kg} / \mathrm{m}^{3}$ and Young's modulus $E=31476 \mathrm{MPa}$. According to the procedure described in the previous point, two hit surfaces were computed by solving Eq. (8) with initial conditions: $f(0)=0, f^{\prime}(0)=1$ (normalization), $f^{\prime \prime}(0)=0$ and $f^{\prime \prime \prime}(0)=\alpha$. The first hit surface represents the values of beam displacements at the end point: $\eta_{1}(\omega, \alpha)=d^{0} f(L) / d x^{0}=f(L)$, whereas for the second one the values of the second derivative are related to the bending moment at the end node $\eta_{2}(\omega, \alpha)=d^{2} f(L) / d x^{2}=f^{\prime \prime}(L)$. Both surfaces are plotted in Fig. 1, which also illustrates 

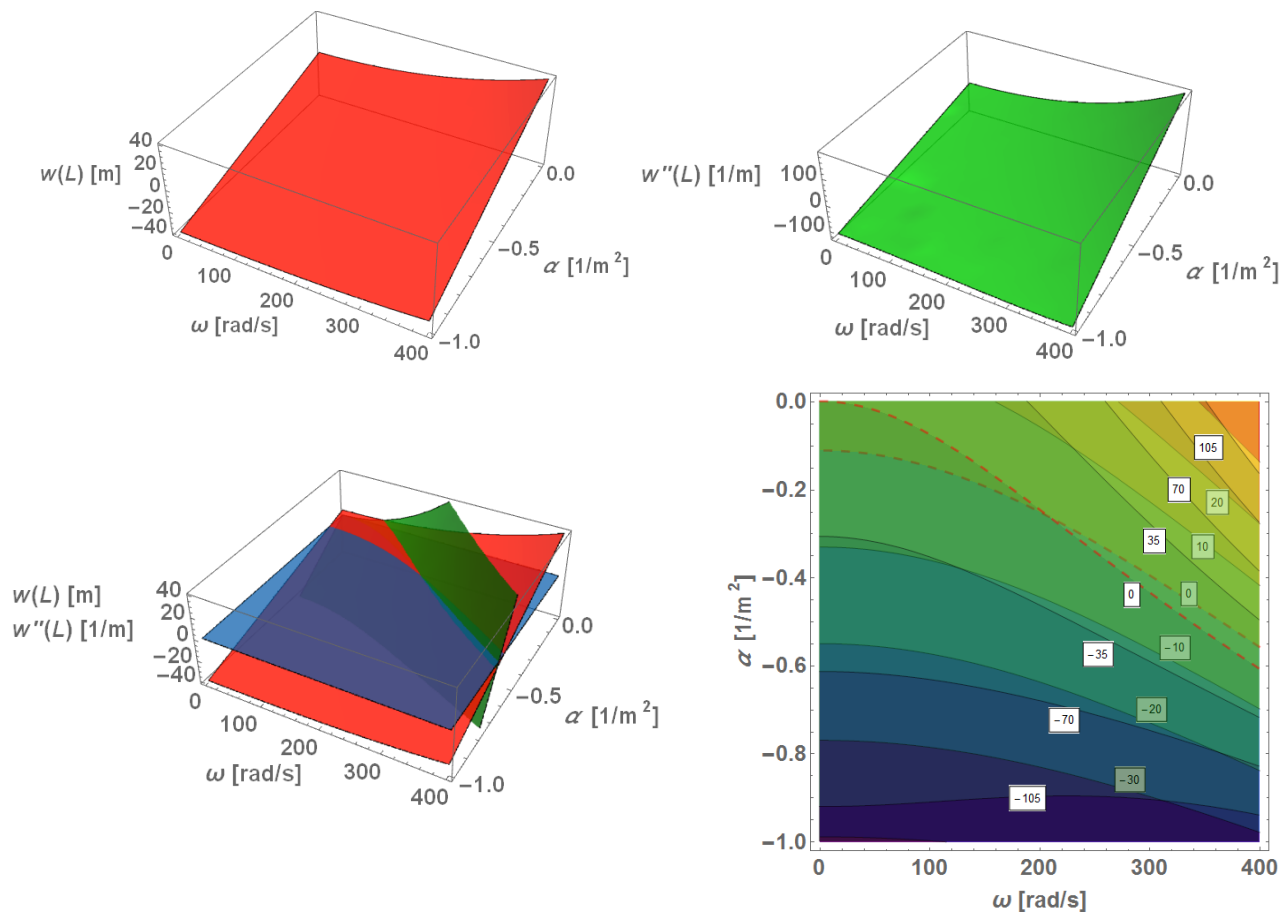

Fig. 1. Hit surfaces obtained by the two-parameter shooting method for the non-prismatic beam.

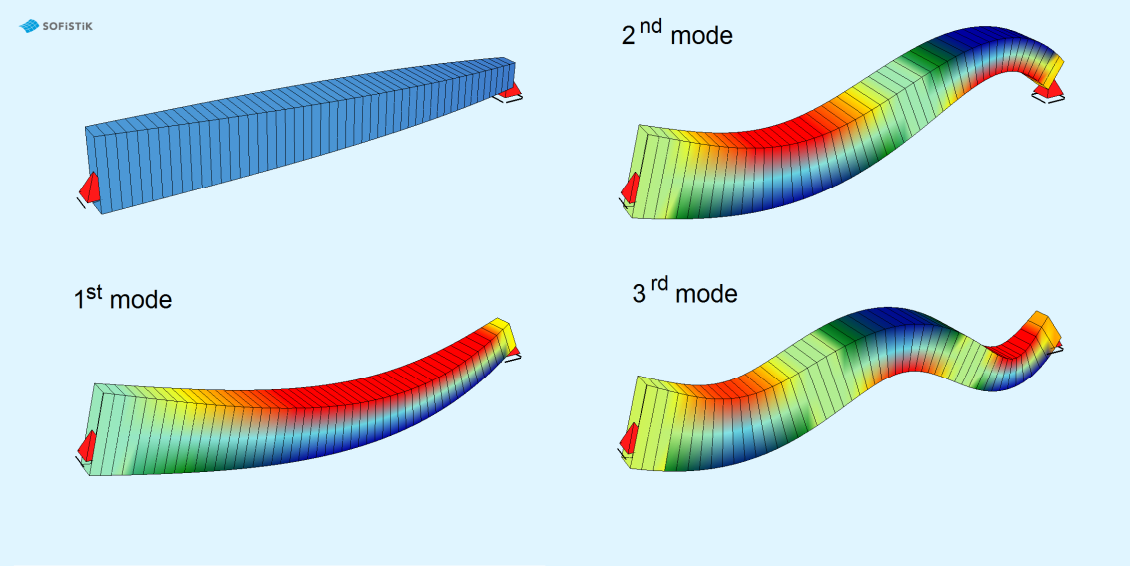

Fig. 2. The non-prismatic concrete beam and three first natural modes calculated in SOFiSTiK.

Table 1. The first three natural frequencies of the non-prismatic beam.

\begin{tabular}{|c|c|c|c|}
\hline \multirow{2}{*}{$\begin{array}{c}\text { Mode } \\
\text { no. }\end{array}$} & \multicolumn{2}{|c|}{ Angular frequency $\omega$ [rad/s] } & \multirow{2}{*}{$\begin{array}{c}\text { Relative } \\
\text { error [\%] }\end{array}$} \\
\cline { 2 - 3 } & Mathematica & SOFiSTiK & 1.41 \\
\hline 1 & 207.50 & 204.57 & 1.32 \\
\hline 2 & 856.89 & 845.61 & 1.15 \\
\hline 3 & 1933.15 & 1910.99 & \multicolumn{2}{|c}{} \\
\hline
\end{tabular}



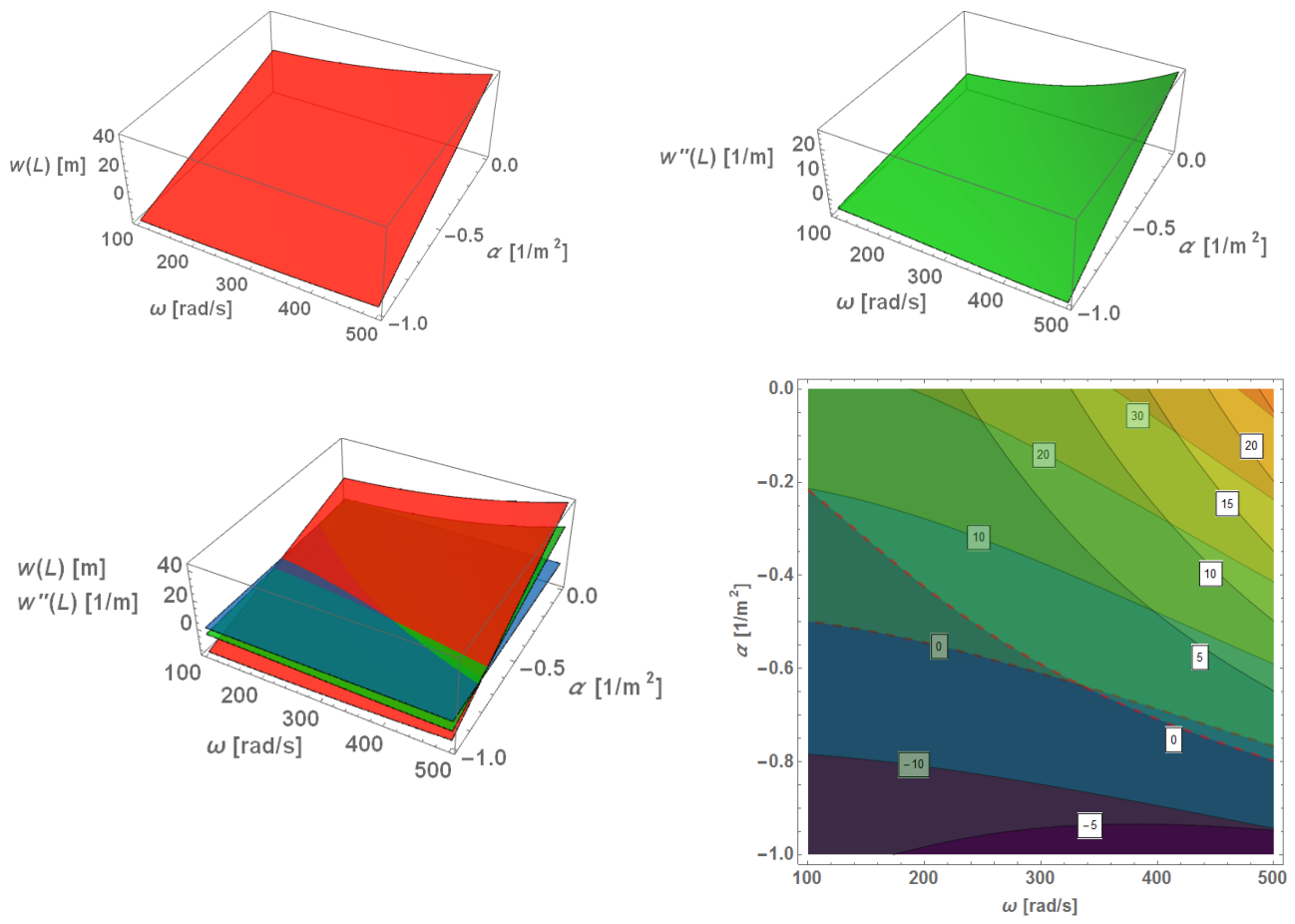

Fig. 3. Hit surfaces obtained by the two-parameter shooting method for the prismatic beam.
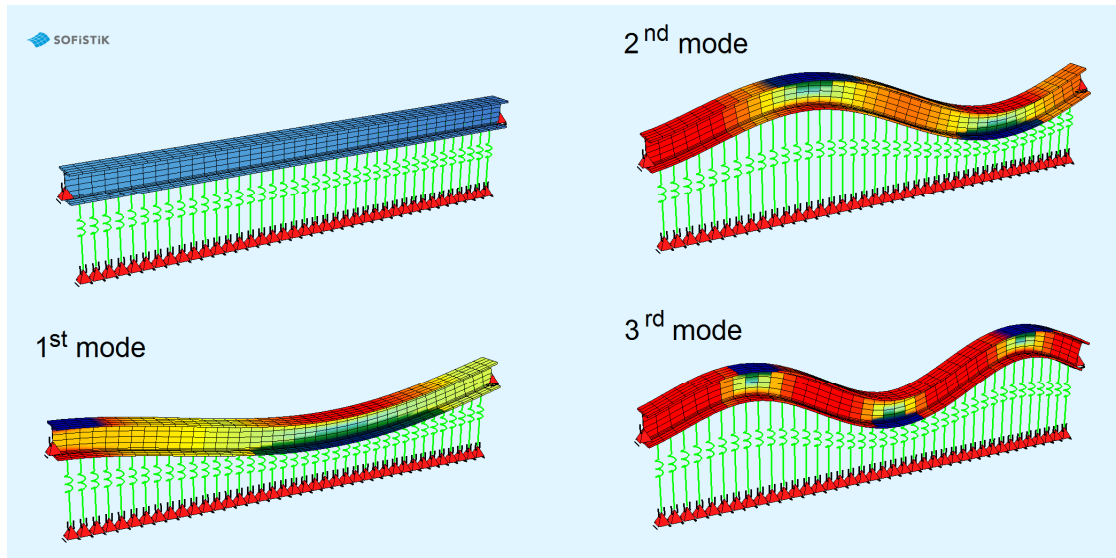

Fig. 4. The prismatic steel beam and three first natural modes calculated in SOFiSTiK.

Table 2. The first three natural frequencies of the prismatic beam.

\begin{tabular}{|c|c|c|c|}
\hline \multirow{2}{*}{$\begin{array}{c}\text { Mode } \\
\text { no. }\end{array}$} & \multicolumn{2}{|c|}{ Angular frequency $\boldsymbol{\omega}[\mathrm{rad} / \mathbf{s}]$} & \multirow{2}{*}{$\begin{array}{c}\text { Relative } \\
\text { error [\%] }\end{array}$} \\
\cline { 2 - 3 } & Mathematica & SOFiSTiK & 0.06 \\
\hline 1 & 335.25 & 335.45 & 0.22 \\
\hline 2 & 1100.85 & 1103.30 & 0.49 \\
\hline 3 & 2306.18 & 2317.46 & \multicolumn{2}{|c}{} \\
\hline
\end{tabular}


the process of solving the system of equations (9). The first intersection point of the two dashed zero contour lines indicates the value of the first natural frequency of the beam.

Further frequencies, not shown in the Fig. 1, are listed in Table 1. To verify the solutions obtained by the two-parameter single shooting method, the same beam was modelled in SOFiSTiK (see Fig. 2). After dividing the structure into 50 finite elements, eigenvalue analysis was performed using the Lanczos method [8]. The computed frequencies and modes are presented in Table 1. As we can see, the results differ slightly, and the maximum relative error does not exceed $1.5 \%$.

\subsection{Prismatic beam subjected to axial force}

In the second example the propped cantilever double T-beam resting on the Winkler foundation with the stiffness parameter $k=100 \mathrm{kN} / \mathrm{m}^{2}$ is considered. The beam with a rolled HEB 360 cross-section and span $L=6.0 \mathrm{~m}$ is made of structural steel S 235 with Young's modulus $E=210000 \mathrm{MPa}$ and a density of $\rho=7850 \mathrm{~kg} / \mathrm{m}^{3}$. The compressive point load $P=2500 \mathrm{kN}$ is applied to the end node of the beam. As in the previous example, two hit surfaces (see Fig. 3) were computed by solving Eq. (8) with initial conditions: $f(0)=0$, $f^{\prime}(0)=0, f^{\prime \prime}(0)=1$ and $f^{\prime \prime \prime}(0)=\alpha$. It should be noted that in this case the natural modes are normalized not by the first but the second derivative of the displacement function. The surfaces and their contour plots are presented in Fig. 3. The obtained results are compared in Table 2 with natural frequencies computed by SOFiSTiK (see Fig. 4). A very good agreement is achieved again, which confirms the effectiveness and universality of the proposed method.

\section{Conclusions}

A novel approach of calculating natural frequencies and modes of single-span beams has been presented. The proposed two-parameter single shooting method is based on the fourth order ordinary differential equation and enables complex problems with two unknown parameters to be solved. Therefore, the method can be successfully used in the analysis of prismatic and non-prismatic beams with different support conditions. The performed analyses confirm that the presented method is formulated correctly and can be used for solving many other problems in a new numerical way.

Calculations were carried out using resources provided by the Wroclaw Centre for Networking and Supercomputing (http://wcss.pl), Grant No. 365.

\section{References}

1. D. Zwillinger, Handbook of Differential Equations (Academic Press, San Diego, 1997)

2. M. A. El-Gebeily, B. S. Attili, Computers and Mathematics with Applications 45 (2003)

3. P. F. Pai, A. N. Palazotto, Int. J. Solid Structures 33, 9 (1996)

4. S. N. Ha, Computers and Mathematics with Applications 42 (2001)

5. S. M. Ibrahim, B. P. Patel,Y. Nath, Int. Journal of Non-Linear Mechanics 44 (2009)

6. P. Ribeiro, Computers and Structures 82 (2004)

7. W. Glabisz, K. Jarczewska, W. Zielichowski-Haber, Archives of Civil and Mechanical Engineering 13, 1 (2013)

8. K. J. Bathe, Finite Element Procedures (Prentice-Hall, New Jersey, 1996) 